\title{
ARTíCUlO
}

\section{Ecología trófica de siete especies de batoideos (Batoidea) en el Pacífico Central Mexicano}

\author{
Trophic ecology of seven batoids species (Batoidea) of the Mexican Central Pacific
}

\begin{abstract}
Juan Ramón Flores-Ortega ${ }^{1,2 *}$, Enrique Godínez-Domínguez² y Gaspar González-Sansón²
${ }^{1}$ Escuela Nacional de Ingeniería Pesquera, Universidad Autónoma de Nayarit. Apartado Postal No ${ }^{\circ}$, Bahía de Matanchén Km 12, San Blas, Nayarit, México. C. P. 63740

${ }^{2}$ Departamento de Estudios para el Desarrollo Sustentable de Zonas Costeras, Universidad de Guadalajara, V. Gómez Farías 82, San Patricio-Melaque, Jalisco, 48980, México. *percebes5@gmail.com

Abstract.-The stomach contents of 7 batoids species (Narcine entemedor, Narcine vermiculatus, Rhinobatos glaucostigma, Urolophus halleri, Urotrygon munda, Urotrygon rogersi and Zapteryx xyster) captured on the continental shelf of the central Mexican Pacific were analyzed in order to describe the diet composition, feeding strategy and trophic interactions during 2 hydroclimatic periods. In total, 1400 stomachs of 7 batoids species were analyzed and 29 prey items were identified (grouped in food category: shrimp, crabs, peracarids, stomatopods, cephalopods, fish and polychaetes). The main prey items were the shrimps (Ogyrididae, Penaeidae and Processidae), serpulid polychaete (Serpullidae) and portunid crabs (Portunidae). According to Smith's index values, the 7 batoids species were generalist with a broad trophic spectrum. During the warm season the highest values of the diet overlap were recorded, mainly between species of the same family (Narcinidae, Rhinobatidae and Urotrygonidae). This pattern might be associated with the opportunism and preference to consume the most abundant prey, which reduces competence for food when resource is scarce.
\end{abstract}

Key words: Batoids, feeding habits, trophic interactions, Mexican Central Pacific

Resumen.- Se realizó el análisis de contenido estomacal de 7 especies de batoideos (Narcine entemedor, Narcine vermiculatus, Rhinobatos glaucostigma, Urolophus halleri, Urotrygon munda, Urotrygon rogersi and Zapteryx xyster) capturadas en la plataforma continental del Pacifico Central Mexicano, para describir la composición de la dieta, estrategias alimentarias e interacciones tróficas durante los 2 periodos hidroclimáticos que se presentan en esta zona. En total se analizaron 1400 estómagos de las 7 especies de batoideos en los cuales se identificaron 29 ítems alimentarios (agrupados en las siguientes categorías alimentarias: camarones, cangrejos, peracáridos, estomatópodos, cefalópodos, peces y poliquetos). Los principales ítems alimentarios fueron los camarones (Ogyrididae, Penaeidae y Processidae), poliquetos serpúlidos (Serpullidae) y cangrejos portúnidos (Portunidae). De acuerdo al índice de Smith las 7 especies de batoideos presentan una estrategia alimentaria de tipo generalista ya que presentan un amplio espectro trófico. En la temporada cálida se presentaron los mayores valores del índice de superposición de la dieta, principalmente entre las especies de la misma familia (Narcinidae, Rhinobatidae y Urotrygonidae). Este patrón está asociado con el oportunismo o el aprovechamiento de los recursos alimentarios de mayor abundancia en ambas temporadas, lo cual reduciría la posibilidad de una competencia por alimento cuando este es escaso.

Palabras clave: Batoideos, hábitos alimentarios, interacciones tróficas, Pacífico Central Mexicano

\section{INTRODUCCIÓN}

Las especies de Batoideos son depredadores activos de los organismos que se encuentran en los fondos blandos y suspendidos en la columna de agua, donde la preferencia alimentaria está directamente relacionada con la talla, la morfología del cuerpo y la posición de la boca (Treloar et al. 2007, Barbini et al. 2011). Los batoideos son importantes depredadores de peces e invertebrados (Ebert \& Cowley 2003, Navia et al. 2007, Follesa et al. 2010, Flores-Ortega et al. 2011), desempeñan un papel importante en las redes tróficas costeras, y pueden servir como conexión del flujo de materia y energía entre el ambiente pelágico y demersal (Bizarro et al. 2007).
La alimentación es una de las actividades que logra explicar con detalle el rol de los organismos en el ecosistema, al igual que las interacciones y los cambios en las preferencias tróficas con relación a los procesos biológicos y físicos en las zonas que habitan (Greene 2013, Rombouts et al. 2013).

La partición de recursos ha sido un factor importante en la explotación de los recursos tróficos por parte de las comunidades demersales para evitar la competencia inter e intraespecies, y es apoyada por la dispersión de los organismos en los gradientes abióticos como la salinidad, oxígeno disuelto o la temperatura, profundidad, además 
del sexo de los organismos, las clases de talla (Bizzarro et al. 2007, Lopez-Lopez et al. 2011) o las temporadas del año (San Martín et al. 2006, Flores-Ortega et al. 2011, Schückel et al. 2011).

En el Pacífico mexicano, los estudios ecológicos de las especies de batoideos se han incrementado en décadas recientes debido a la gran cantidad de especies capturadas en las pesquerías de arrastre y la disminución en la abundancia de las poblaciones, ya que las posibles áreas de crianza (organismos juveniles) y de forrajeo (adultos), pueden ser las mismas zonas de pesca de especies como el camarón (Espinoza et al. 2013).

En las costas del Pacífico central mexicano, las mayores abundancias y biomasas de batoideos se encuentran en ambientes costeros entre los 20 y $40 \mathrm{~m}$ de profundidad (González-Sansón et al. 1997, Flores-Ortega et al. 2011). Los estudios realizados en la comunidad de batoideos en esta zona son escasos y están relacionados con la distribución y abundancia de los organismos (FloresOrtega et al. 2011) y la descripción de la dieta (ValadezGonzález et al. 2000, 2001), sin embargo son escasos los estudios que integran el cambio alimentario estacional y sus interacciones tróficas (Flores-Ortega et al. 2011).

El objetivo de este trabajo fue conocer la composición de la dieta, estrategia alimentaria y las interacciones tróficas de 7 especies de batoideos Narcine entemedor Jordan \& Starks, 1985, Narcine vermiculatus Breder, 1928, Rhinobatos glaucostigma Jordan \& Gilbert 1883, Urolophus halleri (Cooper, 1863), Urotrygon munda Gill, 1863, Urotrygon rogersi (Jordan \& Starks 1895) y Zapteryx xyster Jordan \& Evermann, 1896 capturadas en Pacífico central mexicano durante los 2 periodos hidroclimáticos presentes en esta zona.

\section{MATERIALES Y MÉTODOS}

\section{ÁREa de ESTUdio Y COLECTA DE LAS MUESTRAS}

El área de estudio está localizada en el Pacífico central mexicano (PCM), desde Playa El Coco (19¹0'30'N y $\left.104^{\circ} 39^{\prime} 45^{\prime \prime} \mathrm{W}\right)$ hasta la Bahía de Chamela (19³8'06”N y $105^{\circ} 12^{\prime} 30^{\prime \prime} \mathrm{W}$ ) (Fig. 1). Esta región del PCM presenta una convergencia de 2 sistemas de corrientes importantes. De manera general, el patrón de estacionalidad hidroclimático produce cambios en los procesos costeros (Filonov et al. 2000, Kessler 2006), y determina los ciclos estacionales y la recomposición de las comunidades de zooplancton (Franco-Gordo et al. 2001a,b, 2003; AmbrízArreola et al. 2012, Gasca et al. 2012), peces costeros
(Godínez-Domínguez et al. 2000) y macroinvertebrados bentónicos de fondos blandos (Godínez-Domínguez et al. 2009). La temporada cálida o de influencia de la Contra Corriente Norecuatorial (CCNE), se extiende de julio a diciembre y la temporada fría o de influencia de la Corriente de California (CC), de enero a junio.

Las especies de batoideos fueron capturadas en 67 arrastres de pesca tipo camaronero entre octubre 2007 y mayo 2010. Los arrastres se llevaron a cabo por la noche con una duración de $30 \mathrm{~min}$ para cada arrastre a una velocidad promedio de 2 nudos en 3 profundidades (20, 40 y $60 \mathrm{~m}$ ), y cada arrastre fue posicionado con un sistema de posicionamiento global (GPS) registrados a partir de la introducción del freno del winche, hasta el inicio de la recuperación de la red. Los batoideos fueron separados del resto de la captura para identificar las especies y determinarla longitud total (LT en $\mathrm{cm}$ ) y el peso (W en $\mathrm{g}$ ) de cada uno de los organismos. Los estómagos fueron extraídos y congelados para su posterior análisis en el laboratorio. Los estómagos de los batoideos fueron agrupados por especie y temporada del año [temporada cálida de julio a diciembre (C) y temporada fría de enero a junio (F)]. Para cada una de las especies de batoideos se generaron curvas de acumulación de presas por medio de un procedimiento de remuestreo con 100 aleatorizaciones con el programa EstimateS 8.2. (Colwell 2009) con la finalidad de determinar la suficiencia del número de estómagos analizados.

\section{ANÁlisis de las dietas}

Los ítems alimentarios encontrados fueron contados y pesados (g) e identificados al taxón mínimo posible con ayuda de claves especializadas, luego se agruparon en categorías alimentarias para análisis posteriores (camarones, cangrejos, peracáridos, estomatópodos, cefalópodos, peces y poliquetos). Estos ítems alimentarios se cuantificaron de acuerdo con los métodos tradicionales como el Numérico $(\% N=N i / N t$, donde $N i$ es el número de organismos encontrados de una categoría $i$ y $N t$ es el número total de organismos encontrados de todas las categorías); Gravimétrico ( $\% G=G i / G t$, donde $G i$ es el peso de la categoría $i$ y $G t$ es el peso total de todas las categorías encontradas); Frecuencia de Ocurrencia (\%FO $=\mathrm{No} / \mathrm{Ns}$, donde $\mathrm{No}$ es el número de ocurrencias de la categoría $i$ y $N s$ es el número total de estómagos con alimento analizados) y el Índice de Importancia Relativa $(I I R=(\% N+\% G) * \% F O)($ Pinkas et al. 1971) y es expresado en porcentaje (Cortés 1997). 


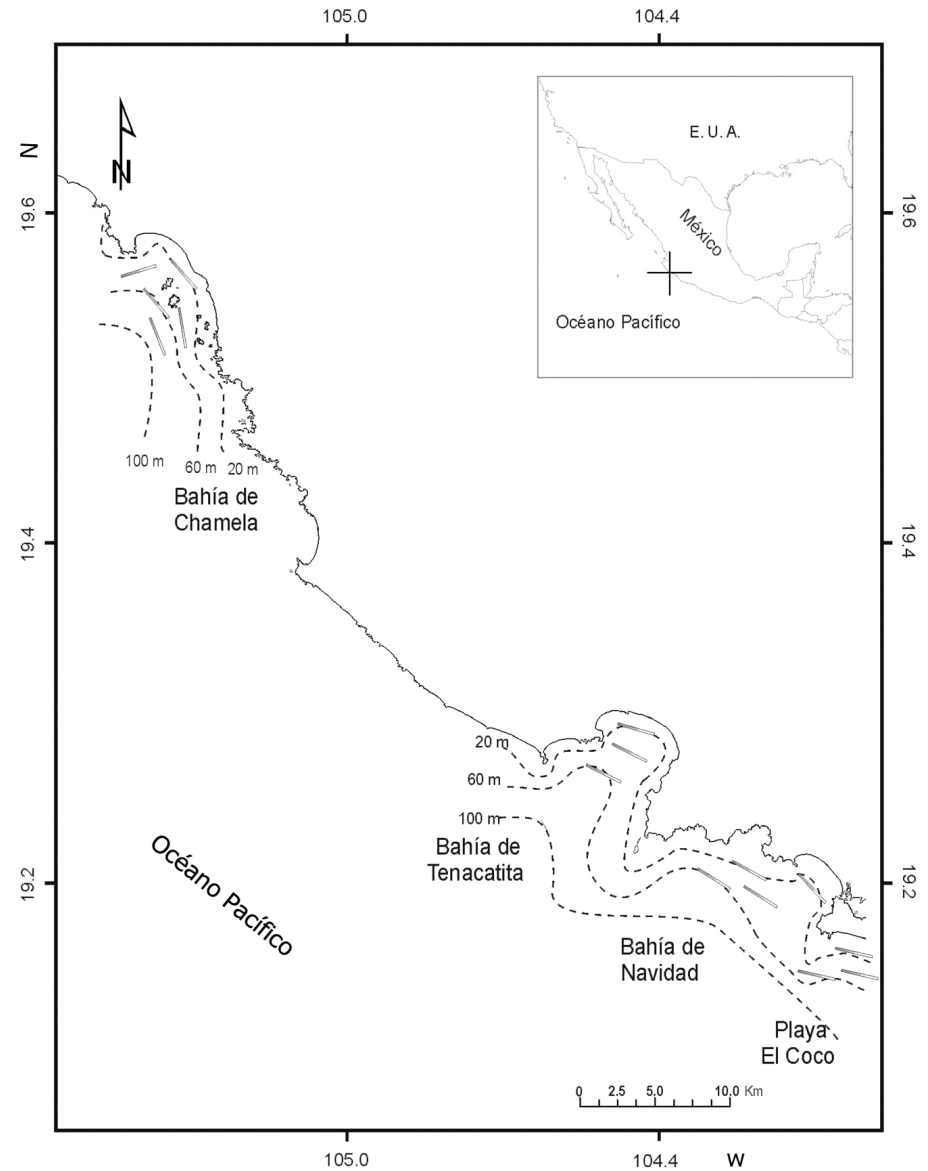

Figura 1. Área de estudio. Las áreas señalan los sitios de captura de las 7 especies de batoideos en el Pacífico central mexicano (PCM) / Study area. The dotted areas indicate the catchsites of the 7 batoids species in the Mexican central Pacific (PCM)
Se estimó el Índice de Smith (1982) para conocer la amplitud del subnicho trófico y la estrategia alimentaria de cada una de las especies de batoideos para cada temporada de estudio, de acuerdo a este índice los valores $<0,6$ las especies son consideradas como especialistas y $\geq 0,6$ se consideran generalistas. Como una medida de la superposición de las dietas entre las especies, se utilizó el índice de Pianka (1973). Cuando los valores oscilan entre el 0 y 0,29 , la superposición es considerada como baja, de 0,3 a 0,6 es considerada media, y mayores de 0,6 la superposición de la dieta se considera alta (Langton 1982). Ambos índices se calcularon en el programa Ecological Methodology 7.2 (Krebs 1999), utilizando los valores porcentuales del índice gravimétrico $(G \%)$ con que contribuye cada uno de los ítems alimentarios en la dieta.

Para analizar el patrón de asociación de presas de acuerdo a la especie por temporada, se utilizaron los métodos de escalado multidimensional no métrico (nMDS, por sus siglas en inglés) y el análisis de clasificación numérica jerárquica aglomerativa (promedio aritmético de grupos de pares no ponderados, UPGMA) (Clarke 1993), ambos estimados con el índice de Bray-Curtis (Clarke \& Warwick 2001) como medida de similitud entre las dietas. Además se estimaron los porcentajes de similitud y disimilitud (SIMPER) para identificar la importancia de los grupos de presas en la dieta de las especies de batoideos en cada temporada (similitud), y entre temporadas (disimilitud). Los análisis se hicieron con el programa PRIMER v6 (Clarke \& Gorley 2006), utilizando los valores porcentuales de índice gravimétrico $(G \%)$ con que contribuye cada uno de los ítems alimentarios en la dieta transformados a raíz cuarta.

Para identificar competencia potencial entre las especies de batoideos se estimó el porcentaje de coocurrencia de captura. Este índice fue calculado con la formula $I C o=N c o / N t$, donde ICo es el Índice de Coocurrencia, $N c o$ es el número de lances en los cuales 2 especies fueron capturadas y $N t$ es el número total de lances en los cuales alguna de las 2 especies fue 
capturada. El valor de co-ocurrencia fue graficado (eje $\mathrm{x}$ ) junto el valor de superposición de la dieta (eje y) de las 2 especies obtenido con el Índice de Pianka (1973) (LopezLopez et al. 2011). Si ambos valores de la interacción entre dos especies se acercan al $100 \%$ demuestran una relación de co-ocurrencia de sitios, una alta superposición de la dieta y una competencia potencial por los recursos en el área de estudio.

\section{Resultados}

\section{DESCRIPCIÓN GENERAL}

Se analizaron en total 1400 estómagos de 7 especies de batoideos de mayor abundancia capturadas en el área de estudio, sin embargo solo en el 74,4\% (1031 estómagos) fueron identificados restos de presas (Tabla 1). En total se identificaron 29 ítems alimentarios en el contenido estomacal de las especies de batoideos analizadas, de las cuales 26 se presentaron en la temporada cálida (C) y 22 en la temporada fría (F) (Tabla 2). Las principales categorías alimentarias en la dieta de las 7 especies de batoideos son los camarones carídeos (Processidae y Ogyrididae), camarones peneidos (Penaeidae), poliquetos serpúlidos (Serpullidae) y cangrejos portúnidos (Portunidae). Las curvas acumuladas de las categorías alimentarias indican que fueron suficientes los estómagos para describir la dieta de $N$. vermiculatus, $U$. halleri, $U$. munda y $U$. rogersi. Sin embargo, para las especies $N$. entemedor, R. glaucostigma y Z. xyster es recomendable incrementar el número de estómagos en el análisis de la dieta de estas 3 especies (Fig. 2).

\section{DESCRIPCIÓN DE LAS DIETAS POR ESPECIE}

En Narcine entemedor fueron analizados 57 estómagos, de los cuales 24 se encontraron contenido. La dieta de esta especie se conformó por 8 ítems alimentarios, de las cuales los camarones alcanzan el $62,5 \%$, los poliquetos el $29 \%$ y los peces el $8,1 \%$ del IIR de la dieta total. Las categorías alimentarias de mayor importancia en la dieta de esta especie son los camarones telescopio (Ogyrididae) (44,9\% IIR), los poliquetos serpúlidos (38,8\% IIR), y los camarones procésidos (Processidae) (5,3\% IIR). Los poliquetos serpúlidos son el alimento principal de $N$. entemedor, ya que alcanzan el 53,8\% durante la temporada cálida y el $82,7 \%$ del IIR durante la temporada fría. Los camarones telescopio y procésidos son las presas que completan la dieta durante la temporada cálida $(20,2$ y $16,5 \%$ del IIR respectivamente) y las esquillas (Squillidae) durante la temporada fría (12\% del IIR).

Se analizaron 209 estómagos para describir la dieta de Narcine vermiculatus, y en 101 estómagos se encontraron contenido. La dieta fue conformada por 13 ítems alimentarios y las categorías alimentarias de los camarones $(67,8 \%$ IIR) y los poliquetos $(31,3 \%$ IIR) son los principales componentes en la dieta. Los camarones telescopio (Ogyrididae) (65,6\% IIR) y los poliquetos serpúlidos (Serpullidae) (32,5\% IIR) son los de mayor importancia en la dieta. En esta especie no se observaron cambios en la composición de la dieta de manera temporal, ya que los camarones telescopio y los poliquetos serpúlidos son las presas de mayor importancia en ambas temporadas de estudio (cálida y fría).

Tabla 1. Número total de estómagos analizados (NT) y con contenido (NC) de las 7 especies de batoideos para cada temporada de estudio / Total number of stomachs analyzed (NT) and stomachs with contents (NC) of the 7 batoids species for each climatic season

\begin{tabular}{lccccc}
\hline \multirow{2}{*}{ Especies } & \multirow{2}{*}{ Abreviaturas } & NT & NC & \multicolumn{2}{c}{ Temporada } \\
\cline { 5 - 6 } N. entemedor & $N e$ & 57 & 24 & 17 & 7 \\
N. vermiculatus & $N v$ & 209 & 101 & 79 & 22 \\
R. glaucostigma & $R g$ & 12 & 10 & 3 & 7 \\
U. halleri & $U h$ & 219 & 196 & 113 & 83 \\
U. munda & $U m$ & 540 & 418 & 256 & 162 \\
U. rogersi & $U r$ & 338 & 265 & 197 & 68 \\
Z. xyster & $Z x$ & 25 & 17 & 11 & 6 \\
& & 1400 & 1031 & 676 & 355 \\
\hline
\end{tabular}




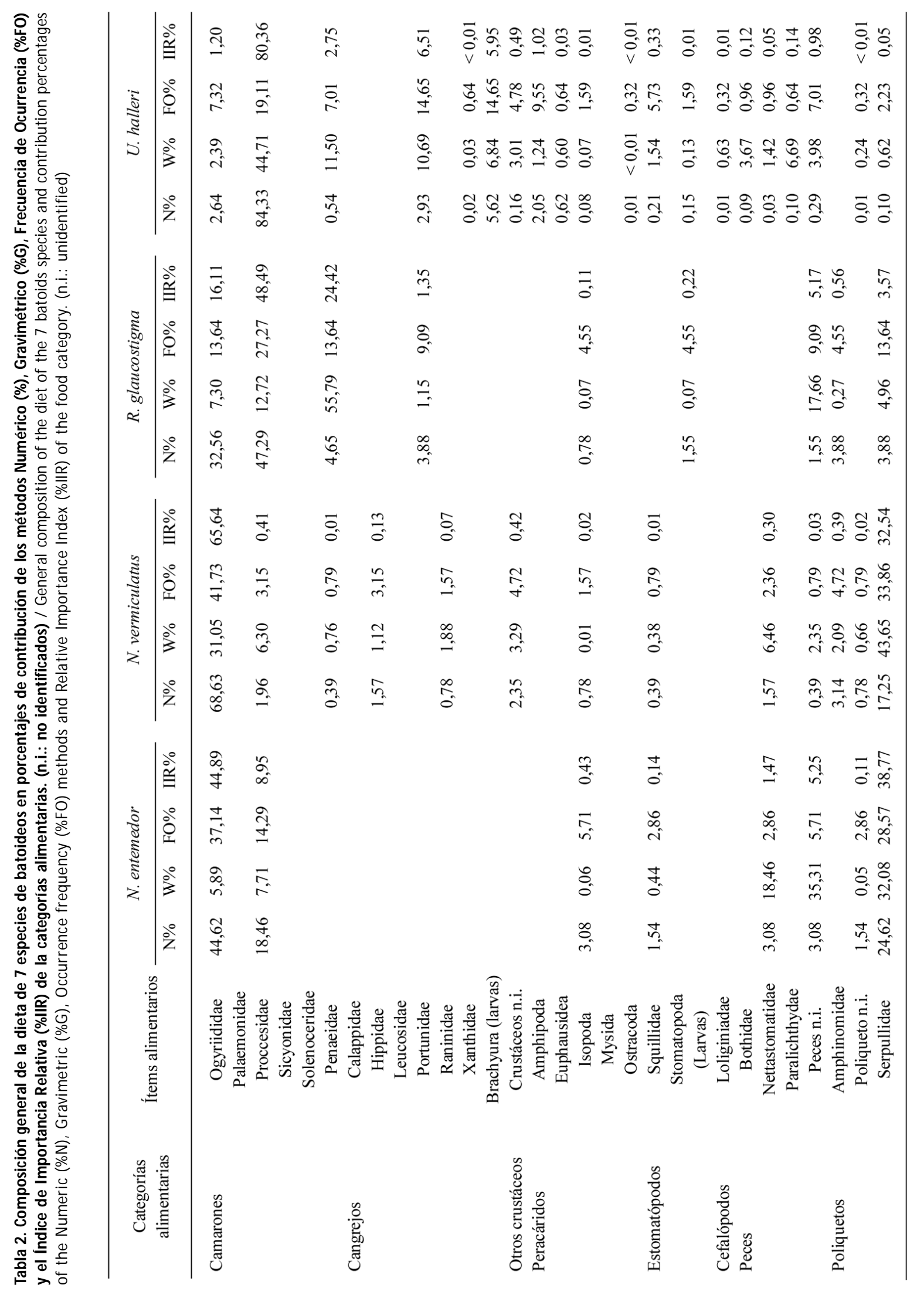


Tabla 2. Continuación / Continued

\begin{tabular}{|c|c|c|c|c|c|c|c|c|c|c|c|c|c|}
\hline \multirow{2}{*}{$\begin{array}{l}\text { Categorías } \\
\text { alimentarias }\end{array}$} & \multirow{2}{*}{$\begin{array}{c}\text { Ítems } \\
\text { alimentarios }\end{array}$} & \multicolumn{4}{|c|}{ U. munda } & \multicolumn{4}{|c|}{ U. rogersi } & \multicolumn{4}{|c|}{ Z. xyster } \\
\hline & & N\% & W\% & $\mathrm{FO} \%$ & IIR\% & N\% & W\% & $\mathrm{FO} \%$ & IIR \% & N\% & W\% & $\mathrm{FO} \%$ & IIR\% \\
\hline \multirow[t]{6}{*}{ Camarones } & Ogyriididae & 31,17 & 22,23 & 23,94 & 29,02 & 33,85 & 17,81 & 22,89 & 31,14 & & & & \\
\hline & Palaemonidae & & & & & 0,03 & 0,21 & 0,26 & $<0,01$ & & & & \\
\hline & Proccesidae & 62,06 & 37,48 & 29,64 & 66,98 & 57,27 & 43,54 & 23,42 & 62,17 & 22,86 & 13,62 & 14,29 & 20,79 \\
\hline & Sicyonidae & 0,03 & 0,37 & 0,33 & $<0,01$ & & & & & 11,43 & 9,47 & 9,52 & 7,94 \\
\hline & Solenoceridae & 0,04 & 0,45 & 0,16 & $<0,01$ & & & & & & & & \\
\hline & Penaeidae & 0,75 & 4,37 & 3,26 & 0,38 & 0,94 & 7,71 & 5,26 & 1,20 & 25,71 & 54,81 & 14,29 & 45,88 \\
\hline \multirow[t]{7}{*}{ Cangrejos } & Calappidae & 0,08 & 1,04 & 0,98 & 0,02 & 0,06 & 0,12 & 0,26 & $<0,01$ & 2,86 & 0,20 & 4,76 & 0,58 \\
\hline & Hippidae & 0,05 & 1,63 & 0,65 & 0,02 & 0,03 & 0,07 & 0,26 & $<0,01$ & & & & \\
\hline & Leucosidae & 0,01 & 0,09 & 0,16 & $<0,01$ & 0,03 & $<0,01$ & 0,26 & $<0,01$ & & & & \\
\hline & Portunidae & 0,59 & 2,89 & 4,23 & 0,33 & 0,97 & 1,65 & 6,32 & 0,44 & & & & \\
\hline & Raninidae & 0,07 & 0,49 & 0,81 & 0,01 & 0,03 & 0,14 & 0,26 & $<0,01$ & & & & \\
\hline & Xanthidae & & & & & & & & & & & & \\
\hline & $\begin{array}{l}\text { Brachyura } \\
\text { (larvas) }\end{array}$ & 0,48 & 0,24 & 2,77 & 0,05 & 0,79 & 0,47 & 3,95 & 0,13 & & & & \\
\hline Otros crustáceos & Crustáceos n.i. & 0,27 & 3,68 & 3,26 & 0,29 & 0,47 & 6,28 & 4,21 & 0,75 & 8,57 & 8,58 & 9,52 & 6,51 \\
\hline \multirow[t]{5}{*}{ Peracáridos } & Amphipoda & 1,14 & 0,39 & 2,28 & 0,08 & 0,85 & 0,47 & 2,89 & 0,10 & & & & \\
\hline & Euphausidea & & & & & & & & & & & & \\
\hline & Isopoda & 0,13 & 0,02 & 0,98 & $<0,01$ & 0,15 & 0,02 & 1,32 & 0,01 & 5,71 & 0,07 & 9,52 & 2,20 \\
\hline & Mysida & 0,01 & 0,01 & 0,16 & $<0,01$ & & & & & & & & \\
\hline & Ostracoda & & & & & & & & & & & & \\
\hline \multirow[t]{2}{*}{ Estomatópodos } & Squillidae & 0,52 & 2,45 & 5,54 & 0,37 & 0,88 & 4,90 & 5,26 & 0,80 & 2,86 & 1,54 & 4,76 & 0,83 \\
\hline & $\begin{array}{c}\text { Stomatopoda } \\
\text { (Larvas) }\end{array}$ & 0,82 & 2,58 & 4,89 & 0,38 & 0,44 & 1,90 & 3,42 & 0,21 & 11,43 & 2,86 & 19,05 & 10,86 \\
\hline Cefalópodos & Loliginiadae & 0,01 & 2,22 & 0,16 & 0,01 & & & & & & & & \\
\hline \multirow[t]{4}{*}{ Peces } & Bothidae & 0,36 & 1,71 & 0,98 & 0,05 & 0,03 & 0,15 & 0,26 & $<0,01$ & & & & \\
\hline & Nettastomatidae & 0,12 & 1,54 & 1,30 & 0,05 & 0,29 & 2,61 & 2,11 & 0,16 & & & & \\
\hline & Paralichthydae & 0,09 & 2,91 & 0,81 & 0,06 & & & & & & & & \\
\hline & Peces n.i. & 0,72 & 8,27 & 8,14 & 1,66 & 1,00 & 6,49 & 7,37 & 1,45 & 2,86 & 8,79 & 4,76 & 2,21 \\
\hline \multirow[t]{3}{*}{ Poliquetos } & Amphinomidae & 0,01 & 0,31 & 0,16 & $<0,01$ & 0,03 & 0,03 & 0,26 & $<0,01$ & & & & \\
\hline & Poliqueto n.i. & 0,13 & 0,38 & 0,65 & 0,01 & 0,23 & 0,51 & 1,58 & 0,03 & & & & \\
\hline & Serpullidae & 0,31 & 2,25 & 3,75 & 0,22 & 1,64 & 4,91 & 8,16 & 1,41 & 5,71 & 0,07 & 9,52 & 2,20 \\
\hline
\end{tabular}

En Rhinobatos glaucostigma se descartaron 2 de los 12 estómagos analizados por encontrase vacíos y se encontraron 9 ítems alimentarios, y la categoría alimentaria de los camarones alcanzó el 95,7\% del IIR total de la dieta. Las principales categorías alimentarias son camarones procésidos (Processidae) (48,5\% IIR), camarones telescopio (Ogyrididae) (16,1\% IIR), y camarones peneidos (Penaeidae) $(24,4 \%$ IIR). En la temporada cálida los poliquetos serpúlidos y los camarones procésidos son los de mayor importancia en la dieta $(76,5$ y $9 \%$ del IIR), y durante la temporada fría los camarones procésidos, peneidos y telescopios son la base alimentaria $(49,0,29,6$ y $13,3 \%$ del IIR respectivamente).

En Urolophus halleri fueron analizados en total 219 estómagos, de los cuales en 196 se encontraron contenido. La dieta de esta especie se conformó de 20 ítems alimentarios, las categorías alimentarias de los camarones (84,9\% IIR) y los cangrejos (11,7\% IIR) son los principales componentes de la dieta. Los camarones procésidos (Processidae) (80,4\% IIR), los cangrejos portúnidos (Portunidae) (6,5\% IIR), y las larvas de braquiuros (6\% IIR) son los ítems alimentarios de mayor importancia en la dieta. Los camarones procésidos (86,97\% IIR) y las larvas de braquiuros $(6,4 \%$ IIR) son los principales ítems alimentarios de mayor importancia en la dieta durante la temporada cálida y en cambio durante la temporada fría los cangrejos portúnidos (38,3\% IIR), los anfípodos (Amphipoda) (16,3\% IIR), los camarones telescopio (Ogyrididae) (12,3\% IIR) y los lenguados de tallas pequeñas (Paralichthydae) $(11,1 \%$ IIR) son los que conforman principalmente la dieta. 

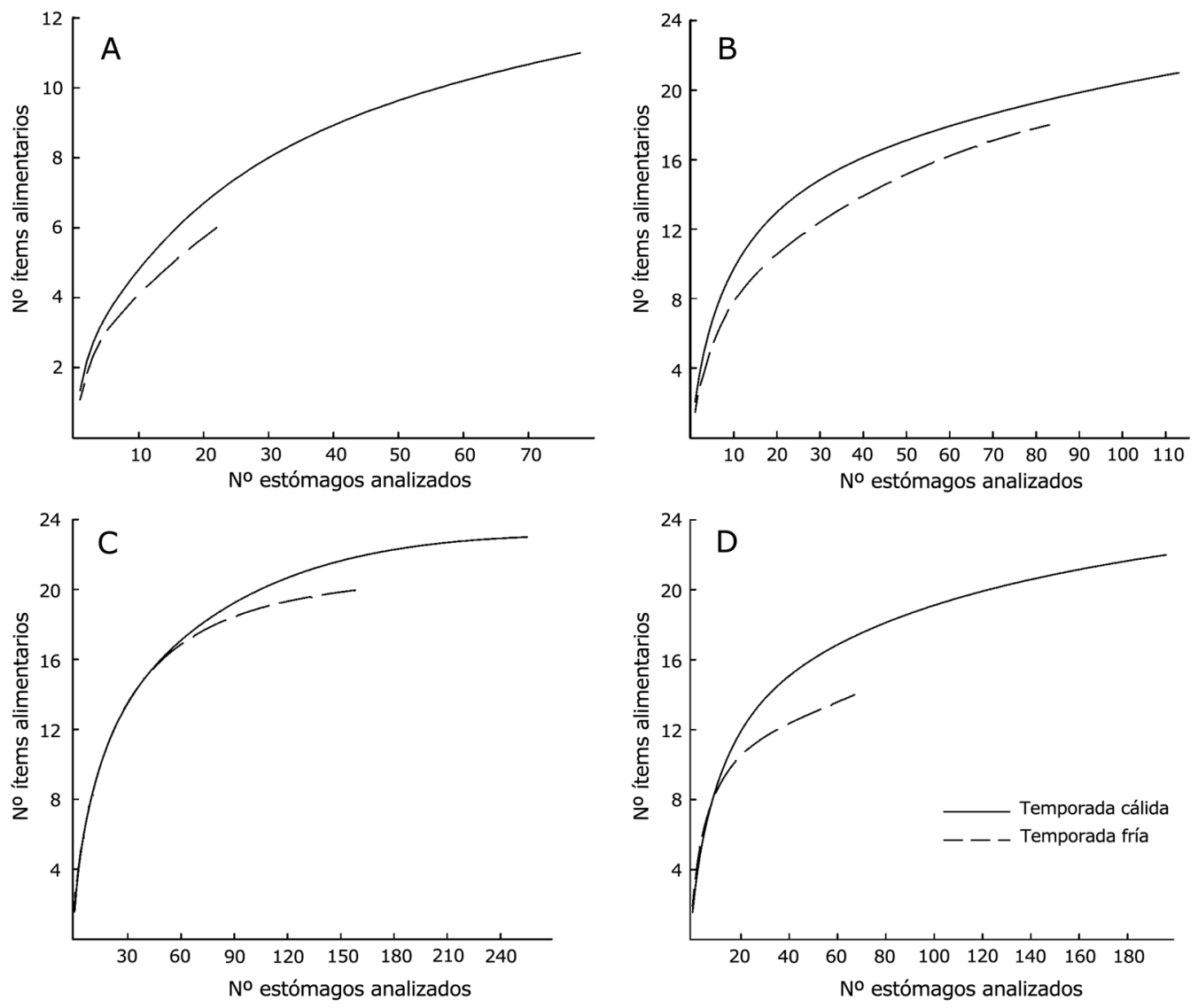

Figura 2. Curvas acumuladas de los ítems presa encontradas en los estómagos de las 4 especies de batoideos generadas al azar con 100 remuestreos. A) N. vermiculatus, B) U. halleri, C) U. munda, D) U. rogersi / Cumulative prey items curves found in the stomach of the 7 batoids species randomly generated with 100 resamplings. A) N. vermiculatus, B) U. halleri, C) U. munda, D) U. rogersi

Para describir la dieta de Urotrygon munda fueron analizados 540 estómagos, de los cuales solo en 418 estómagos se encontraron con alimento. La dieta se conformó por 25 ítems alimentarios, los camarones $(96,7 \%$ IIR) y los peces $(1,7 \%$ IIR) son las principales categorías alimentarias que componen la dieta de esta especie. Los camarones procésidos (Processidae) (67\% IIR) y los camarones telescopio (Ogyrididae) (29\% IIR) son las de mayor importancia en la dieta. En ambas temporadas los camarones procésidos son los ítems alimentarios de mayor importancia en la dieta, con el 77,9\% del IIR durante la temporada cálida y el 52,1\% del IIR durante la temporada fría.

De los 338 estómagos analizados de Urotrygon rogersi, 265 se encontraban con contenido. La dieta de esta especie la conformaron 21 ítems alimentarios, de los cuales los camarones $(96,7 \%$ IIR) y los peces $(1,1 \%$ IIR) son las principales categorías alimentarias en la dieta de esta especie. Los camarones procésidos (Processidae) $(62,2 \%$ IIR) y los camarones telescopio (Ogyrididae) (31,1\% IIR), son las presas de mayor importancia al tener más del $93 \%$ del IIR total de la dieta. Durante la temporada cálida los camarones procésidos son la principal pres en la dieta (71,5\% IIR) y los camarones telescopio durante la temporada fría $(59,95 \%$ IIR).

Se analizaron 25 estómagos de Zapteryx xyster para describir su dieta, de los cuales en 17 estómagos se encontró contenido. La dieta de esta especie se conformó de 10 ítems alimentarios y las categorías de mayor importancia en la dieta son los camarones (92,2\% IIR) y los estomatópodos (5,6\% IIR). Los camarones peneidos (Penaeidae) (45,9\% IIR), los camarones procésidos (Processidae) (20,8\% IIR), las larvas de estomatópodos (10,9\% IIR) y los camarones siciónidos (Sicyonidae) $(7,9 \%$ 
IIR). Los camarones siciónidos (34,5\% IIR), las larvas de estomatópodos (35,3\% IIR) son los ítems alimentarios de mayor importancia en la dieta en la temporada cálida los camarones peneidos y procésidos $(73,4$ y $23,2 \%$ IIR respectivamente), en la temporada fría.

\section{ESTRATEGIA ALIMENTARIA, SUPERPOSICIÓN DE DIETAS Y CO-OCURRENCIA ALIMENTARIA}

Las 7 especies de batoideos presentan una estrategia alimentaria de tipo generalista y un amplio espectro trófico en la dieta (Fig. 3), ya que de acuerdo al índice de Smith los valores obtenidos son superiores a $0,6(0,64-0,79)$. En lo que refiere a la superposición de la dieta de las 7 especies de batoideos, los mayores valores de superposición se presentaron durante la temporada cálida, y entre especies de la misma familia. En la familia Narcinidae los valores de superposición de la dieta osciló entre 0,62 y 0,92, entre las 2 especies de la familia Rhinobatidae fue de 0,95 , y entre las especies de la familia Urotrygonidae el valor de superposición osciló entre 0,85 y 0,97.

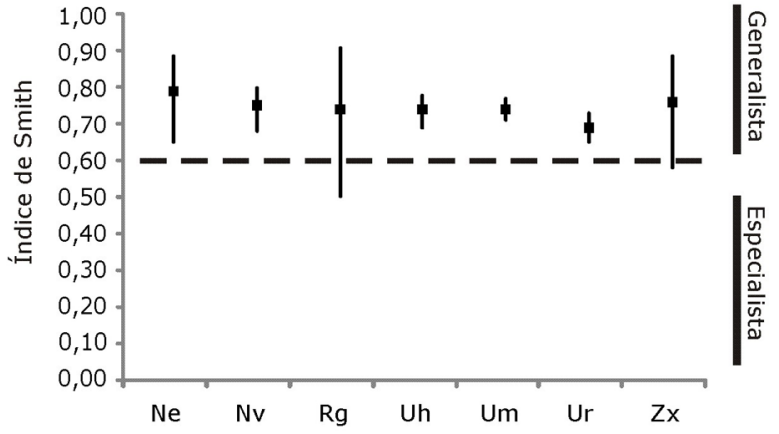

Figura 3. Valores promedio del Índice de Smith $( \pm 95 \%$ intervalo de confianza) para las 7 especies de batoideos analizadas. (Abreviaturas en la Tabla 1) / Smith's index mean values ( $\pm 95 \%$ confidence interval) for the 7 batoids species analyzed. (Abbreviations in Table 1)

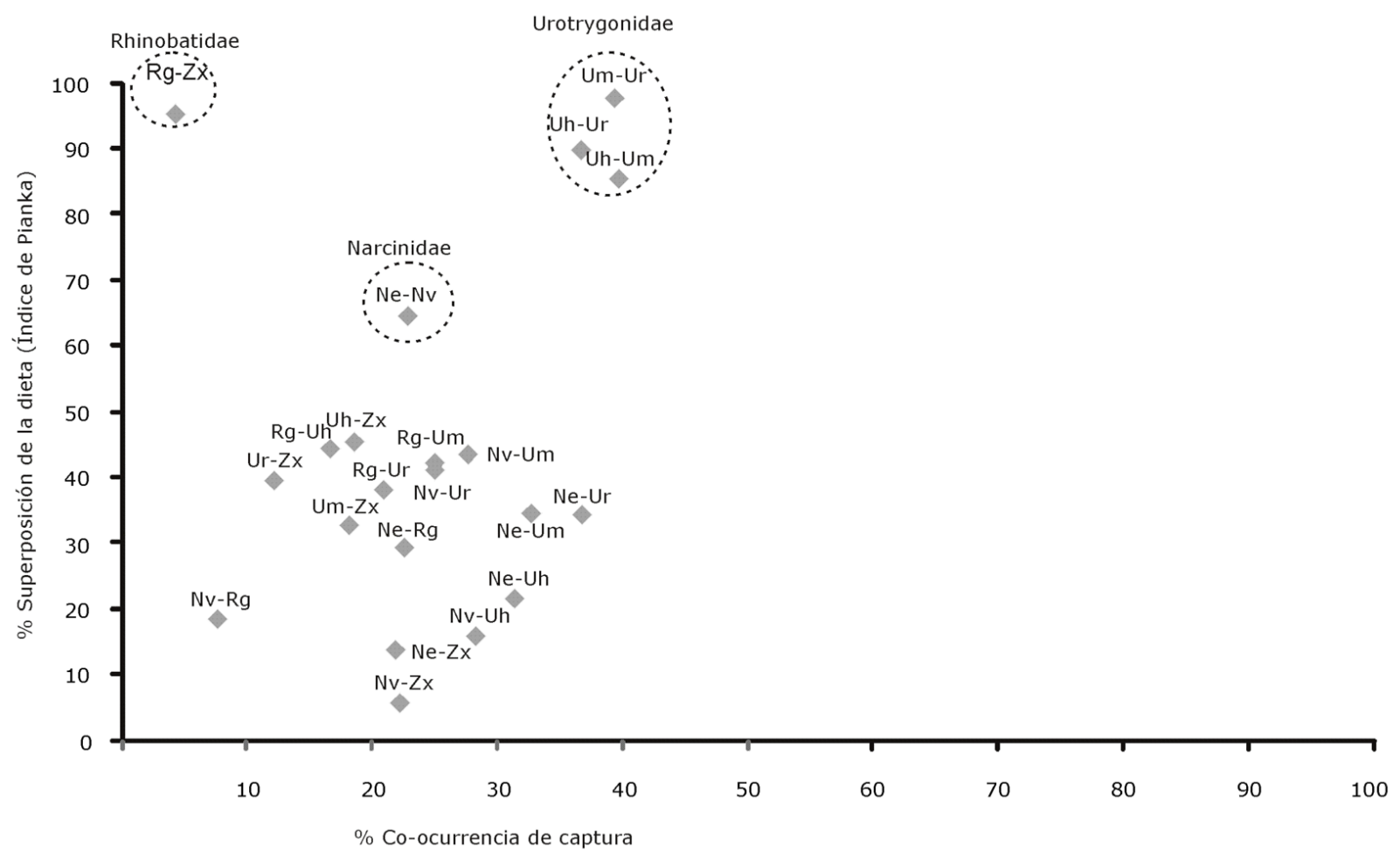

Figura 4. Valores porcentuales de co-ocurrencia de captura (eje $x$ ) y del índice de superposición de dieta de Pianka (eje y) entre las 7 especies de batoideos analizadas. (Abreviaturas en la Tabla 1) / Percentage values of co-occurrence capture (axis $\mathrm{x}$ ) and diet overlap of Pianka's index value (axis y) between 7 batoids species analyzed. (Abbreviations in Table 1) 
La probabilidad más alta de capturar 2 especies de batoideos en un mismo lance en el área de estudio está por abajo del $40 \%$ (Fig. 4), lo que disminuye la posibilidad de que los organismos coincidan al alimentarse en un sitio y tiempo determinado. Los valores más altos del Índice de Co-ocurrencia lo presentaron las especies de la familia Urotrygonidae (40\%).

De acuerdo a los valores de similitud de Bray-Curtis y el nMDS se observó un patrón de asociación estacional entre las especies de batoideos en relación a la dieta (Figs. 5 y 6), y la partición de los recursos entre las especies de estos depredadores demersales, fue establecida de acuerdo a la similitud taxonómica entre las especies analizadas y la formación de grupos funcionales en la dieta.

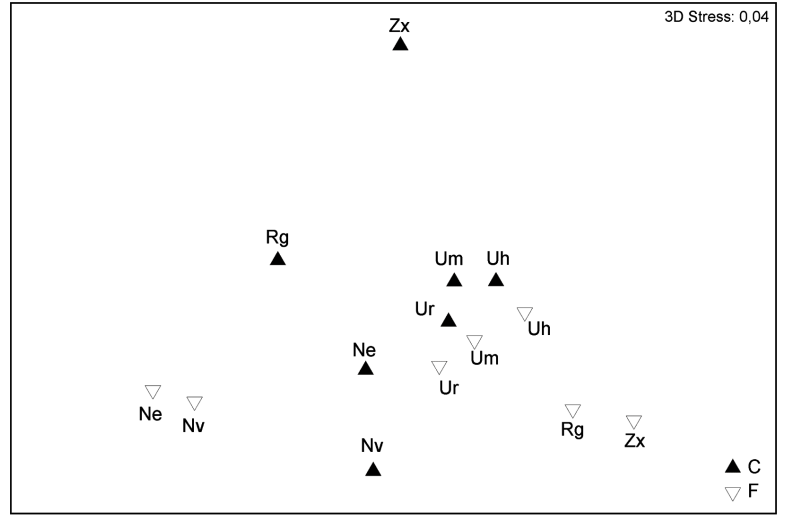

Figura 5. Escalado multidimensional no métrico de las 7 especies de batoideos por temporada de estudio ( $C=$ Cálida y F= Fría). (Abreviaturas en Tabla 1) / Non-metric multidimensional scaling of the 7 batoids species analyzed for study season $(C=W$ arm and $\mathrm{F}=$ Cold). (Abbreviations in Table 1)

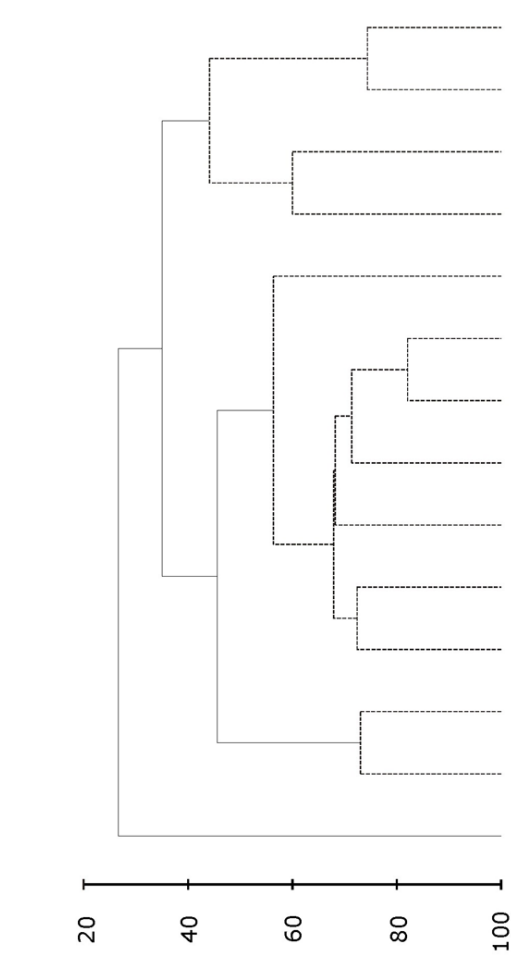

$\%$ Similitud (Bray-Curtis)

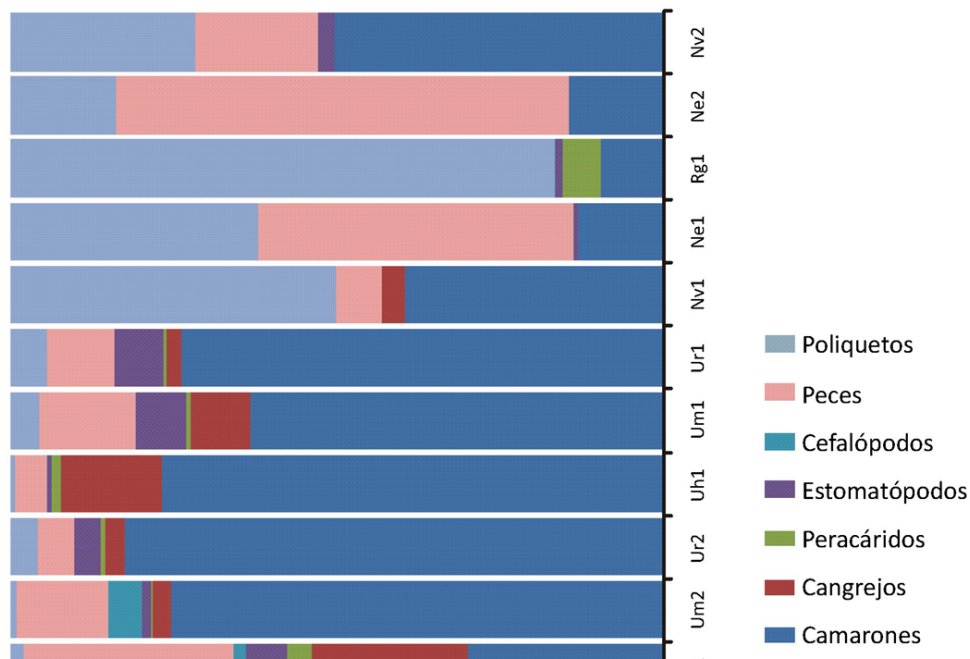

Figura 6. Análisis de clasificación numérica jerárquica aglomerativa (UPGMA) y composición de la dieta (categorías alimentarias) de las 7 especies de batoideos por temporada de estudio ( $C=$ Cálida y F= Fría). (Abreviaturas en Tabla 1) / Numerical classification of agglomerative hierarchical analysis (UPGMA) and diet composition (food categories) of the 7 batoids species by study season $(C=W a r m$ and $F=C o l d)$. (Abbreviations in Table 1) 
Tabla 3. Análisis SIMPER de los ítems alimentarios encontrados en las 7 especies de batoideos para cada temporada de estudio / SIMPER analysis of the prey items found in the 7 batoids species for each study season

\begin{tabular}{lccc}
\hline \multicolumn{1}{c}{$\begin{array}{c}\text { Ítems } \\
\text { alimentarios }\end{array}$} & \multicolumn{3}{c}{ Temporadas } \\
\cline { 2 - 4 } & Cálida & Fría & $\begin{array}{c}\text { Cálida } v s \\
\text { Fría }\end{array}$ \\
\hline Processidae & 20,2 & 13,4 & 3,8 \\
Serpullidae & 17,9 & 8,3 & 4,6 \\
Ogyriididae & 11,9 & 23,6 & 3,6 \\
Peces n.i. & 7,8 & 11,7 & 3,6 \\
Crustáceos n.i. & 7,6 & & 3,2 \\
Squillidae & 5,9 & 4,9 & 2,7 \\
Stomatopoda & 5,7 & & 3,2 \\
(Larvas) & 4,7 & & 1,7 \\
Isopoda & 3,4 & 13,9 & 4,6 \\
Penaeidae & 3,0 & 10,3 & 3,9 \\
Nettastomatidae & 2,6 & & 2,1 \\
Amphipoda & & 4,4 & 2,7 \\
Portunidae & & & 1,9 \\
Sicyonidae & & & 1,7 \\
Brachyura & & & 1,7 \\
larvas & & & 1,7 \\
Poliqueto n.i. & & & 1,6 \\
Bothidae & & & \\
Paralichthydae & & & \\
\hline & & & \\
\hline
\end{tabular}

El análisis de similitud de dietas entre las especies de batoideos en ambas temporada de estudio muestra que los poliquetos $(28,9 \%$ en la cálida y $15,1 \%$ en la fría) y los camarones telescopio ( $15,6 \%$ en cálida y $20,5 \%$ en fría) y procésidos (16,5\% en cálida y 14,6\% en fría), son los ítems alimentarios que tipifican la dieta, al igual que la disimilitud en las dietas entre las temporadas con el 11,1, 8,9 y $8,8 \%$ (Poliquetos, Ogyrididae y Processidae, respectivamente) (Tabla 3 ).

\section{Discusión}

El patrón alimentario de las 7 especies de batoideos analizadas es similar a lo reportado para otras especies de batoideos alrededor del mundo (Navia et al. 2007, Treloar et al. 2007, Barbini et al. 2011, Payan et al. 2011), y lo reportado en el PCM (Valadez-González et al. 2000, 2001; Flores-Ortega et al. 2011). Debido a la forma y posición de la boca, los batoideos tienen la preferencia por consumir presas bentónicas que se encuentran en los sedimentos marinos como poliquetos, crustáceos, moluscos y peces (Kobelkowsky 2013). Se ha descrito a las especies de batoideos como consumidoras de pequeños invertebrados como camarones, cangrejos, poliquetos y peces de pequeño tamaño, sin importar zonas geográficas ni intervalos batimétricos de distribución (Barbini et al. 2010, Flores-Ortega et al. 2011, De la RosaMeza et al. 2013, Espinoza et al. 2013). En los fondos blandos de zonas tropicales se concentra una alta riqueza, diversidad y abundancia de macroinvertebrados y peces que son la base alimentaria para abundantes depredadores (Corgos et al. 2014), tales como los batoideos que habitan el fondo marino o simplemente lo utilizan como un sitio de alimentación (Link et al. 2002, Ruiz-Rodríguez et al. 2013).

En las costas del PCM se ha reportado un patrón estacional de desplazamiento de la asociación de macroinvertebrados bentónicos, ya que durante la temporada cálida la mayor abundancia y biomasa se localizan a los 60-80 m de profundidad, y durante la temporada fría se desplazan hacia la costa $(20-40 \mathrm{~m})$ (Godínez-Domínguez \& González-Sansón 1998), en cambio las especies de batoideos muestran las mayores abundancias en torno a $\operatorname{los} 20 \mathrm{~m}$ de profundidad $\mathrm{sin}$ presentar cambios estacionales importantes (FloresOrtega et al. 2011). La segregación de los depredadores en el gradiente batimétrico o cambios en las preferencias alimentarias relacionadas con los cambios ontogénicos han sido la clave en la diversificación de las estrategias alimentarias entre los organismos y así evitar la competencia entre los organismos (Ebert \& Cowley 2003, Barbini et al. 2011, Payan et al. 2011, López-García et al. 2012), lo cual no ha sido observado como una característica en la comunidad de peces demersales estudiados en el PCM (Flores-Ortega 2013, Flores-Ortega et al. 2011, 2013).

Los depredadores con estrategias generalistas y/o oportunistas tienen la capacidad de cambiar el tipo de alimento en respuesta a la disponibilidad y abundancia de recursos alimentarios en el medio (Rinewalt et al. 2007). En la gran mayoría de estudios tróficos realizados a las especies de batoideos se les ha categorizado como depredadores generalistas, aunque es importante mencionar que este generalismo alimentario está estrechamente relacionado con el oportunismo de consumir y aprovechar los recursos disponibles y de mayor abundancia a lo largo del año (San Martín et al. 2006, Flores-Ortega et al. 2011). 
Los cambios en la disponibilidad y abundancia de presas en el medio natural han llevado a las especies de rayas, lenguados y otras especies de peces, a adoptar una estrategia alimentaria de tipo generalista oportunista, ya que las variaciones inter e intraespecies en la composición de la dieta de los organismos reduce la posibilidad de una competencia potencial por el alimento (Sommerville et al. 2011). Las especies de la familia Urotrygonidae presentan un cambio en la dieta de acuerdo a las temporadas y sitios de alimentación, relacionado además con la abundancia y disponibilidad de las presas (Flores-Ortega et al. 2011, Navia et al. 2011). La amplitud del nicho trófico para las especies de batoideos analizadas en este trabajo coincide ampliamente con lo descrito por otros autores para estas especies en otras latitudes, lo que conlleva a destacar la facilidad de adaptación de las especies de batoideos a los cambios en los tipos de presas que pueden consumir (De la Rosa-Meza et al. 2013, Espinoza et al. 2013).

En el PCM la diversidad y disponibilidad de los recursos alimentarios (especies forrajes según Blaber et al. 1995) en ambas temporadas de estudio (Corgos et al. 2014) contribuye para que las especies de batoideos desarrollen una generalización de los subnichos tróficos, lo cual reduce considerablemente la superposición de la dieta y minimiza la posibilidad de establecer una competencia por el alimento en los fondos blandos.

\section{Agradecimientos}

JRFO agradece al Consejo Nacional de Ciencia y Tecnología de México (CONACyT) por el apoyo financiero durante los estudios de posgrado. Los autores agradecemos a los revisores anónimos por sus comentarios para mejorar este estudio.

\section{LITERATURA CITADA}

Ambriz-Arreola I, J Gómez-Gutiérrez, C Franco-Gordo, BE Lavaniegos \& E Godínez-Domínguez. 2012. Influence of coastal upwelling-downwelling variability on tropical euphausiid abundance and community structure in the inshore Mexican central Pacific. Marine Ecology Progress Series 451: 119-136.

Barbini SA, LB Scenna, DE Figueroa, MB Cousseau \& JM Díaz de Astarloa. 2010. Feeding habits of the Magellan skate: effects of sex, maturity stage, and body size on diet. Hydrobiologia 641(1): 275-286.

Barbini SA, LO Lucifora \& NM Hozbor. 2011. Feeding ecology and habitat selectivity of the Zapteryx brevirostris (Chondrichthyes, Rhinobatidae) off north Argentina and Uruguay. Marine Biology Research 7: 365-377.
Bizzarro JJ, HJ Robinson, CS Rinewalt \& DA Ebert. 2007. Comparative feeding ecology of four sympatric skate species off central California, USA. Environmental Biology of Fishes 80: 197-220.

Blaber SJM, DT Brewer \& JP Salini. 1995. Fish communities and the nurseryrole of the shallow inshore waters of a tropical bay in the Gulf of Carpentaria, Australia. Estuarine Coastal and Shelf Science 40: 177-193.

Clarke KR. 1993. Non-parametric multivariate analyses of changes in community structure. Australian Journal of Ecology 18: 117-143.

Clarke KR \& RN Gorley. 2006. PRIMER v6: User Manual/ Tutorial, 192 pp. PRIMER-E, Plymouth Marine Laboratory, Plymouth.

Clarke KR\& RM Warwick. 2001. Change in marine communities: an approach to statistical analysis and interpretation, $144 \mathrm{pp}$. PRIMER-E, Plymouth Laboratory, Plymouth.

Colwell RK. 2009. EstimateS: Statistical estimation of species richness and shared species from samples. Version 8.2. <http:/ /purl.oclc.org/estimates>

Corgos A, E Godínez-Domínguez, JR Flores-Ortega \& JA Rojo-Vázquez. 2014. Comunidades de fondos blandos submareales de la bahía de Navidad (Jalisco, México): patrones espaciales y temporales. Revista Mexicana de Biodiversidad 85: 1171-1183.

Cortés E. 1997. A critical review of methods of studying fish feeding based on analysis of stomach contents: application to elasmobranch fishes. Canadian Journal of Fisheries and Aquatic Science 54: 726-738.

De la Rosa-Meza K, O Sosa-Nishizaki \& H De la CuevaSalcedo. 2013. Feeding habits of the speckled guitarfish Rhinobatos glaucostigma (Elasmobranchii, Batoidea) in the southeastern Gulf of California. Ciencias Marinas 39(3): 277290.

Ebert DA \& PD Cowley. 2003. Diet, feeding behaviour and habitat utilisation of the blue stingray Dasyatis chrysonota (Smith, 1828) in South African waters. Marine Freshwater Research 54: 957-965.

Espinoza M, TM Clarke, F Villalobos-Rojas \& IS Wehrtmann. 2013. Diet composition and diet feeding behaviour of the banded guitarfish Zapteryx xyster along the Pacific coast of Costa Rica, Central America. Journal of Fish Biology 82(1): 286-305.

Filonov AE, IE Tereshchenko, CO Monzón, ME GonzálezRuelas \& E Godínez-Domínguez. 2000. Variabilidad estacional de los campos de temperatura y salinidad en la zona costera de los estados de Jalisco y Colima, México. Ciencias Marinas 26: 303-321.

Flores-Ortega JR. 2013. Ecología trófica de la asociación de peces demersales de mayor abundancia en el Pacífico Central Mexicano. Tesis Doctoral, Centro Universitario de la Costa Sur, Universidad de Guadalajara, San Patricio-Melaque, 119 pp. 
Flores-Ortega JR, E Godínez-Domínguez, G GonzálezSansón, JA Rojo-Vázquez, A Corgos \& MY MoralesJáuregui. 2011. Feeding habits of three round stingrays (Rajiformes: Urotrygonidae) in the central Mexican Pacific. Ciencias Marinas 37(3): 279-292.

Flores-Ortega JR, E Godínez-Domínguez, G GonzálezSansón, JA Rojo-Vázquez, A Corgos \& MY MoralesJáuregui. 2013. Abundancia, distribución, hábitos alimentarios e interacciones tróficas de cinco especies de lenguados (Pleuronectiformes) en el Pacífico central mexicano. Latin American Journal of Aquatic Research 41(3): 423-439.

Follesa MC, A Mulas, S Cabiddu, C Porcu, AM Deiana \& A Cau. 2010. Diet and feeding habits of two skate species, Raja brachyuran and Raja miraletus (Chondrichthyes, Rajidae) in Sardinian waters (central-western Mediterranean). Italian. Journal of Zoology 77(1): 53-60.

Franco-Gordo C, E Godínez-Domínguez \& E SuárezMorales. 2001a. Zooplankton Biomass variability in the Mexican Eastern tropical Pacific. Pacific Science 55: 191202.

Franco-Gordo C, E Suárez-Morales, E Godínez-Domínguez \& R Flores-Vargas. 2001b. A seasonal survey of the fish larvae community of the central Pacific coast of México. Bulletin of Marine Science 68: 383-396.

Franco-Gordo C, E Godínez-Domínguez \& E SuárezMorales. 2003. Zooplancton diversity in the central Mexican Pacific. Estuarine, Coastal Shelf Science 57: 111-121.

Gasca R, C Franco-Gordo, F Godínez-Domínguez \& E Suárez-Morales. 2012. Hyperiid amphipod community in the Eastern Tropical Pacific before, during, and after El Niño 1997-1998.Marine Ecology Progress Series 455: 123-139.

Godínez-Domínguez E \& G González-Sansón. 1998. Variación de los patrones de distribución batimétrica de la fauna macrobentónica en la plataforma continental de Jalisco y Colima, México. Ciencias Marinas 24: 337-351.

Godínez-Domínguez E, JA Rojo-Vázquez, VH Galván-Piña \& B Aguilar-Palomino. 2000. Changes in the structure of a coastal fish assemblage exploited by a small scale gillnet fishery during an El Niño-La Niña event. Estuarine, Coastal and Shelf Science 51:773-787.

Godínez-Domínguez E, J Freire, C Franco-Gordo \& G González-Sansón. 2009. Decomposing diversity patterns of a soft-bottom macroinvertebrate community in the tropical eastern Pacific. Journal of the Marine Biological Association of the United Kingdom 89(1): 31-38.

González-Sansón G, B Aguilar-Palomino, J ArciniegaFlores, R García de Quevedo-Machaín, E GodínezDomínguez, V Landa-Jaime, J Mariscal-Romero, JE Michel-Morfín\& M Saucedo-Lozano. 1997. Variación espacial de la abundancia de la fauna de fondos blandos en la plataforma continental de Jalisco y Colima, México (Primavera 1995). Ciencias Marinas 23(1): 93-110.
Greene CH. 2013. Towards a more balanced view of marine ecosystems. Fisheries Oceanography 22: 140-142.

Kessler WS. 2006. The circulation of the eastern tropical Pacific: A review. Progress in Oceanography 69: 181-217.

Kobelkowsky A. 2013. Morfología del sistema digestivo de la raya mariposa Gymnuramicrura (Batoidea: Gymnuridae). Boletín de Investigaciones Marinas y Costeras 42(1): 57-71.

Krebs CJ. 1999. Ecological methodology, 620 pp. Benjamin Cummings, California.

Langton RW. 1982. Diet overlap between the Atlantic cod Gadus morhua, silver hake Merluccius bilinearis and fifteen other northwest Atlantic finfish. Fishery Bulletin 80: 745-759.

Link JS, K Bolles \& CG Milliken. 2002. The feeding ecology of flatfish in the Northwest atlantic. Journal of Northwest Atlantic Fishery Science 30: 1-17.

López-García J, AF Navia, PA Mejia-Falla \& EA Rubio. 2012. Feeding habits and trophic ecology of Dasyatis longa (Elasmobranchii: Myliobatiformes): sexual, temporal and ontogenetic effects. Journal of Fish Biology 80(5): 15631579.

Lopez-Lopez L, I Preciado, F Velasco, I Olaso \& JL GutiérrezZabala. 2011. Resource partitioning amongst five coexisting species of gurnards (Scorpaeniforme: Triglidae): Role of trophic and habitat segregation. Journal of Sea Research 66(2): 58-68.

Navia AF, PA Mejía-Falla \& A Giraldo. 2007. Feeding ecology of elasmobranch fishes in coastal waters of the Colombian Eastern Tropical Pacific. BMC Ecology 7: 8. <doi:10.1186/ 1472-6785-7-8>

Payan LF, AF Navia, EA Rubio \& PA Mejía-Falla. 2011. Biologia de la raya guitarra Rhinobatos leucorhynchus (Günther, 1867) (Rajiformes: Rhinobatidae) en el Pacifico colombiano. Latin American Journal of Aquatic Research 39(2): 286-296.

Pianka ER. 1973. The structure of lizard communities. Annual Review of Ecology, Evolution and Systematics 4: 53-74.

Pinkas L, MS Oliphant \& LK Iverson. 1971. Food habits of albacore, bluefin tuna, and bonito in Californian waters. California Department of Fish and Game, Fish Bulletin 152: $1-105$.

Rinewalt CS, DA Ebert \& GM Cailliet. 2007. Food habits of the sandpaper skate, Bathyraja kincaidii (Garman, 1908) off central California: seasonal variation in diet linked to oceanographic conditions. Environmental Biology of Fishes 80: 147-163.

Rombouts I, G Beaugrand, X Fizzala, F Gaill, SPR Greenstreet, S Lamare, F Le Loc'h, A McQuattersGollop, B Mialet, N Niquil, J Percelay, F Renaud, AG Rossberg \& JP Féral. 2013. Food web indicators under the Marine Strategy Framework Directive: From complexity to simplicity? Ecological Indicators 29: 246-254. 
Ruiz-Rodríguez T, AR Vázquez-Bader \& A Gracia-Gasca. 2013. Asociaciones de megacrustáceos epibentónicos en la Sonda de Campeche, Golfo de México. Revista Mexicana de la Biodiversidad 84: 280-290.

San Martin MJ, JM Braccini, LL Tamini, GE Chiaramonte \& JE Perez. 2006. Temporal and sexual effects in the feeding ecology of the marbled sand skate Psammobatis bergi Marini, 1932. Marine Biology 151(2): 505-513.

Smith EP. 1982. Niche breadth, resource availability, and inference. Ecology 63: 1675-1681.

Sommerville E, ME Platell, WT White, AA Jones \& IC Potter. 2011. Partitioning of food resources by four abundant, co-occurring elasmobranch species: relationships between diet and both body size and season. Marine and Freshwater Research 62: 54-65.
Treloar MA, LJB Laurenson \& JD Stevens. 2007. Dietary comparisons of six skate species (Rajidae) in south-eastern Australian waters. Environmental Biology of Fishes 80(23): 181-196.

Valadez-González C, B Aguilar-Palomino \& S HernándezVázquez. 2000. Dieta de la raya Narcine entemedor en la plataforma continental de Jalisco y Colima, México. Boletín del Centro de Investigaciones Biológicas 34(1): 1-20.

Valadez-González C, B Aguilar-Palomino \& S HernándezVázquez. 2001. Hábitos alimentarios de la raya Urobatis halleri (Cooper, 1863) (Chondrichthyes: Urolophidae) capturada en la plataforma continental de Jalisco y Colima, México. Ciencias Marinas 27: 91-104.

Recibido el 07 de enero 2015 y aceptado el 12 de agosto de 2015

Editor: Claudia Bustos D. 